Case Report

\title{
Primary Leptomeningeal Lymphoma Presenting as Low Back Pain and Dementia: A Case Report
}

\author{
Vida Margarette de Vera Andal ${ }^{1,}$, , Julette Marie Feliciano Batara ${ }^{1}$, Joven Racpan Cuanang ${ }^{2}$ \\ ${ }^{1}$ Department of Neurosciences, University of the Philippines, Philippine General Hospital, Manila, Philippines \\ ${ }^{2}$ St. Luke's Medical Center Global City, Taguig, Philippines \\ Email address: \\ vida_andal@yahoo.com (V. M. de V. Andal),jbataral@yahoo.com (J. M. F. Batara), jovencuanang@gmail.com (J. R. Cuanang) \\ ${ }^{*}$ Corresponding author
}

\section{To cite this article:}

Vida Margarette de Vera Andal, Julette Marie Feliciano Batara, Joven Racpan Cuanang. Primary Leptomeningeal Lymphoma Presenting as Low Back Pain and Dementia: A Case Report. Clinical Neurology and Neuroscience. Vol. 2, No. 2, 2018, pp. 33-40.

doi: $10.11648 /$ j.cnn.20180202.13

Received: May 31, 2018; Accepted: July 4, 2018; Published: July 30, 2018

\begin{abstract}
Primary leptomeningeal lymphoma (PLL) is an extremely rare condition, with only a handful of cases reported in literature. Most often, these are misdiagnosed as other disease entities that are more commonly observed in practice. Currently, there are no clinical algorithms that may guide clinicians to clinching a diagnosis of PLL. This paper discusses a remarkable case of PLL presenting with atypical symptoms namely dementia and low back pain. This is a case of an apparently well adult male who initially presented with low back pain that was treated for two years as a case of lumbar disc herniation. This developed into lower extremity weakness and was subsequently followed by dementia and seizures. The patient was initially treated for tuberculous meningitis after CSF findings showed lymphocytic predominance. However, lack of clinical improvement prompted further work up revealing a malignant process in the lumbosacral meninges. Dural biopsy showed atypical mononuclear infiltrates consistent with a B-cell non-Hodgkin lymphoma with immunohistochemistry showing CD20 positive, CD79a positive, CD3 negative. Being MUM1 positive and BCL2 positive suggests it being a diffuse large B cell lymphoma. A primary malignancy of the leptomeninges was ruled in after PET CT showed no other sites of high metabolic activity. The patient was able to complete 6 cycles of high dose Methotrexate and three Rituximab doses. Remarkably, after more than two years after his initial presentation, he still has mild signs of dementia but is able to carry out most activities of daily living with minimal assistance. The patient has survived beyond the expected life expectancy for PLL.
\end{abstract}

Keywords: Primary Leptomeningeal Lymphoma, Atypical Back Pain, Dementia

\section{Introduction}

Primary CNS Lymphoma (PCNSL) is a disease wherein malignant lymphocytes and lymphoblasts invade the brain parenchyma and rarely, in the other parts of the CNS. The brain is devoid of lymphatic tissue so the exact mechanism of how malignant lymphoblasts reach the CNS is still a mystery. Some authors propose that it is likely metastasis from systemic lymphoma though most refute this theory because such lymphomas rarely metastasize.

PCNSL used to comprise a negligible portion of all brain tumors. However, in the past two decades, there is almost a triple-fold increase in incidence, accounting to about $3.1 \%$ of all brain tumors [1]. This rise is partially attributed to the concomitant increase of cases of AIDS and other immunosuppressed states. In about $60 \%$ of cases, PCNSL arises from the cerebral hemispheres as solitary or multifocal masses, most commonly in the periventricular areas. In about $10-20 \%$ of cases, it arises from the retina, uvula and vitreous body (intraocular lymphoma). Rarely does it occur primarily in the pia or arachnoid matter. This makes it a challenge to clinch diagnosis in these areas.

Most leptomeningeal lymphomas are metastatic processes from a known parenchymal CNS or systemic lymphoma. Fischer et. al prospectively studied 282 patients diagnosed with primary CNS lymphoma and investigated the incidence of 
leptomeningeal spread through CSF cytomorphology and MRI. It ranged from $16-42 \%$, depending on the technique used in detecting spread. The study, however, did not include cases where leptomeningeal biopsy was needed for diagnosis [2].

Primary Leptomeningeal Lymphoma (PLL) is a rare occurrence, comprising only $7 \%$ of already rare primary CNS lymphomas [3]. The largest case series published about it by Taylor et.al reviewed 48 patients between 1981 and 2011. Most cases presented with multifocal, non-localizing signs and symptoms, ranging from craniopathies, radiculopathies, headaches, ataxia, seizures and encephalopathies [4]. It is quite common to misdiagnose it as stroke, meningitis, meningioma or even polyradiculopathies [5]. In a review by Iwamoto and Abrey, it is observed that is has a female predilection, with a 3-4: 1 female/male ratio. In contrast with PCNSL, PLL has no clear association with immunocompromised states [6].

MRI findings include linear areas of enhancement along the Virchow-Robin spaces, walls of the ventricles and on the roots of the cauda equina. Cerebrospinal fluid (CSF) morphologic assessment is considered as the gold standard in detecting meningeal involvement of carcinomas. However, it fails to detect such in $41 \%$ of cases, most often due to paucity of tumor cells [7]. In such cases, a meningeal biopsy is done to confirm diagnosis. In the case series mentioned earlier, $33 \%$ of patients required biopsy prior to diagnosis. However, it is hard to apply the same to all patients presenting with a constellation of non-specific symptoms.

PLL is usually a diagnosis of exclusion. A proposed diagnostic criteria include (a) absence of systemic lymphoma; (b) no prior organ transplantation, immunosuppressive therapy, systemic or intrathecal antineoplastic drug therapy or acquired immune deficiency syndrome; (c) radiologic findings not consistent with parenchymal or subependymal CNS lesion; (d) negative test for systemic lymphoma; and (e) malignant lymphocytes seen in CSF or meningeal biopsy. [8]

Most cases of PLL are of B-cell type. There are only a few reports of primary T-cell leptomeningeal lymphoma. A small case series reports poorer survival for the T-cell type [9] but the report of the International Primary CNS Lymphoma Collaborative Group showed no significant difference in the median survival rate of T-cell as compared to primary CNS lymphoma in general [10].

There is limited data as to the most effective treatment regimen for PLL. High dose methrotrexate is so far the most effective drug for PCNSL but its efficacy in PLL is unclear [6].

We describe a patient diagnosed with primary leptomeningeal lymphoma who initially presented with back pain and dementia.

\section{Case Report}

WDR is a 62-year-old, married, hypertensive Filipino male who has no prior exposure to toxins or radiation. He owns a business that produces processed meat but he has no direct contact with chemicals used. He is confined to managerial activities and paperwork. He used to smoke 2 packs daily for about 55 years and has only stopped when his symptoms started. He denies alcohol consumption and drug use. He has had decreased hearing acuity in his left ear for years.

He presented two years prior to diagnosis with a vague, dull, non-radiating low back pain which did not affect his activities of daily living. He consulted with an orthopedic surgeon who ordered for a plain lumbosacral MRI. It revealed multilevel degenerative disc disease and disc bulges resulting to varying degrees of spinal canal and neural foraminal stenosis (figure 1).

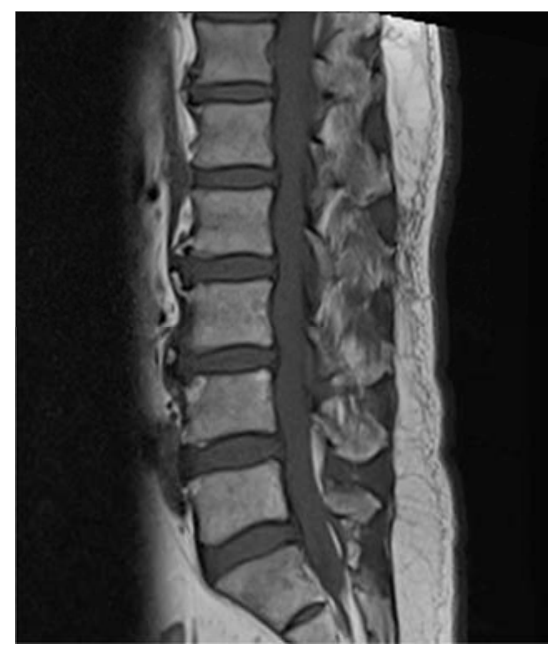

Figure 1. T1 weighted image of patient WDR's lumbosacral spine in sagittal view showing multiple degenerative disc disease and intervertebral disc bulges.

He was diagnosed to have lumbar disc herniation and underwent percutaneous lumbar endoscopic discectomy of L3-L4. Post-operatively, he was still ambulatory, with no weakness or numbness in extremities. He was still able to carry out his normal activities at work and at home but there was no relief of the back pain he initially complained of.

Several months after the operation, his family noticed generalized weakness, loss of appetite and vigor, and subsequent weight loss. A year after his surgery, he complained of lower extremity weakness described as inability to get up the stairs with a feeling of unsteadiness. He also noted some tingling sensation on his distal lower extremities. After a few weeks, the weakness of his lower extremities progressed and he required assistance in ambulation. He preferred to stay in bed or was sometimes wheelchair bound. At this time, he developed urinary retention and constipation.

He sought consult with a neurologist for second opinion. On physical examination, he had intact cranial nerves but with decreased hearing acuity on the left. On muscle motor testing, he scored $4 / 5$ on all movements of the lower extremities. There was areflexia in patellar and ankle reflexes and no extensor toe sign. At this time, a malignant process involving the cauda equina versus an infectious process was entertained. A lumbosacral MRI with contrast was done and it showed hazy appearance of the subarachnoid space and diffuse enhancement of the CSF, as well as the previously noted multilevel disc disease. Because of these findings, a cranial MRI with contrast was then done and it showed moderate ventriculomegaly. 
A lumbar tap was done and it yielded straw, clear CSF with 116 lymphocytes and no neutrophils and no RBC. It had very high glucose at $116 \mathrm{mg} / \mathrm{dl}(120 \%$ of serum) and very high protein at $867 \mathrm{mg} / \mathrm{dl}$. However, CSF AFB, CALAS and bacterial culture studies turned out negative. CSF histopathology showed mature lymphocytes with a background of eosinic amorphous material.

Because of this inconclusive results, a repeat lumbar tap was done. The opening pressure registered $20.15 \mathrm{~cm}$ water. It drained xanthochromic CSF with low flow. It showed RBC of $53,125 \times 10^{6} / \mathrm{L}, \mathrm{WBC}$ of $1,287 \times 10^{6} / \mathrm{L}$ which was $98 \%$ lymphocytes. It had normal glucose at $126 \mathrm{mg} / \mathrm{dl}(53.6 \%$ of serum) and very high protein at 2,608 mg/dl. Again, CSF AFB, bacterial culture studies and CALAS were negative. He was empirically treated for tuberculous meningitis but he only complied for 5 months.

Meanwhile, his gait worsened and he started to lose weight

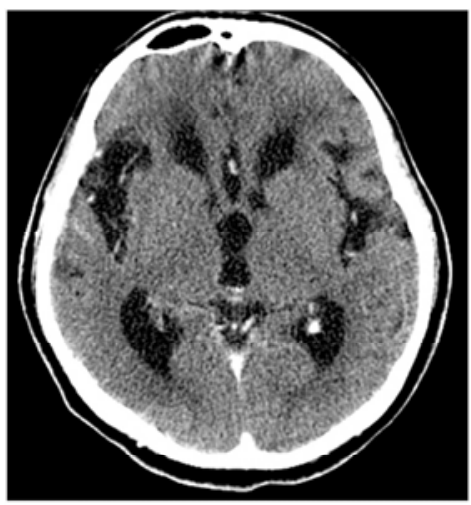

(A)

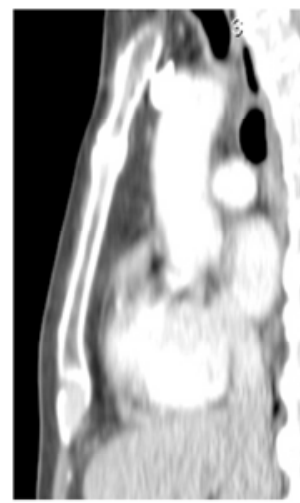

(B) and get depressed. His relatives noted marked decrease in his verbal output, often conversing in phrases or words only. There is an increase in loss of vigor and decrease in level of activity. He often had memory lapses. He easily forgets recent events but can accurately remember things of the past. He also started to have occasional tremors of his distal extremities, lasting only for a few seconds and resolves spontaneously without any loss of consciousness.

Four months later, he developed seizures described as convulsive movement of extremities with head turning to the left. Each episode lasted for around 10 seconds followed by post ictal loss of consciousness. These attacks happened once a month. He sought for another neurologist's opinion. On EEG, he had good baseline activity $(11-13 \mathrm{~Hz})$ and no epileptiform changes were seen. On whole body CT scan with contrast, it showed ventriculomegaly with leptomeningeal enhancement from cranial meninges down to the lumbosacral spine (Figure 2).

and $C)$, most marked on the cauda equina $(C)$.

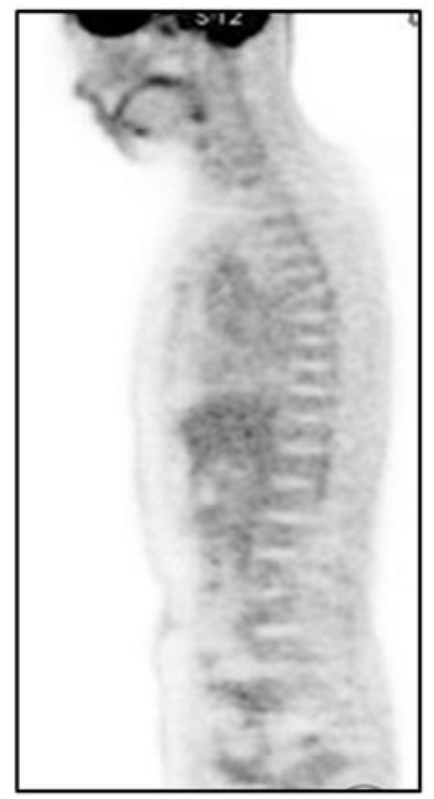

(A)

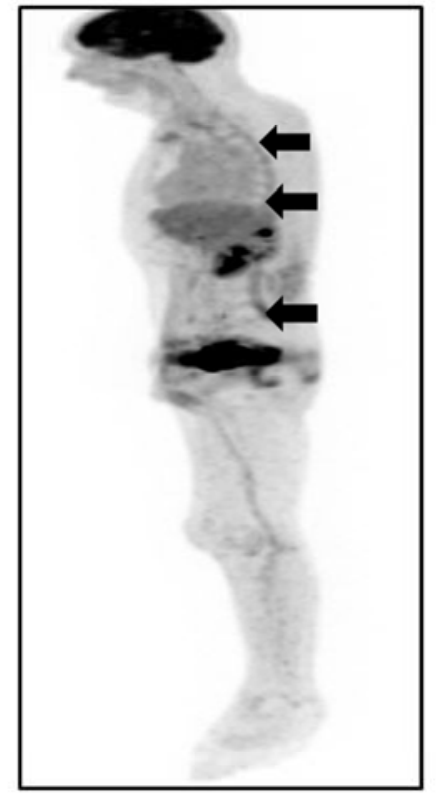

(B)
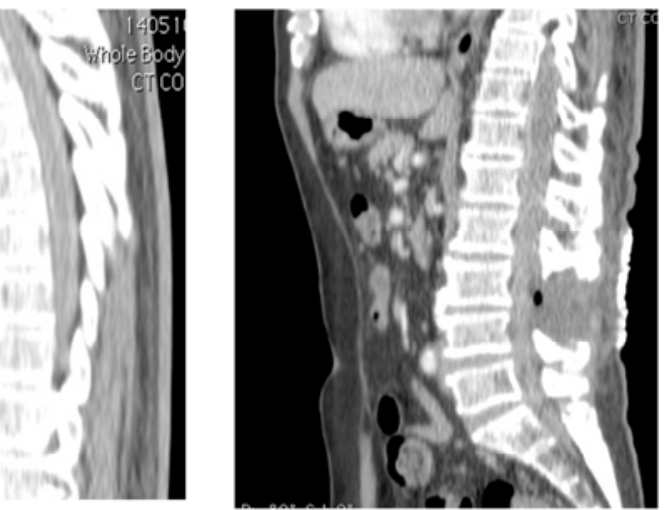

(C)

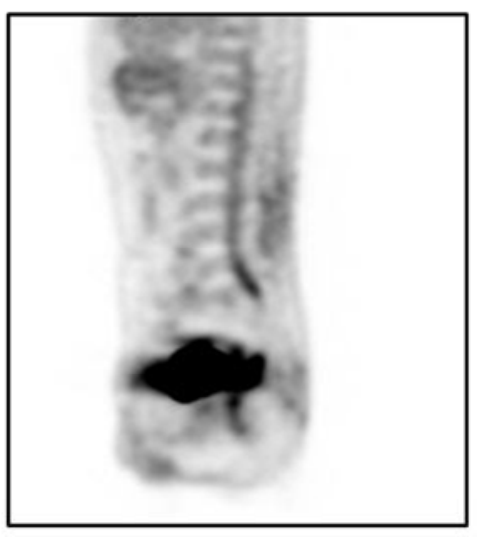

(C)

Figure 3. A normal PET scan (A) showing normal uptake of the spine [11] as compared to patient WDR who has increased metabolic activity from the cervical to the lumbar cord (B), most intense on the cauda equina $(C)$. 


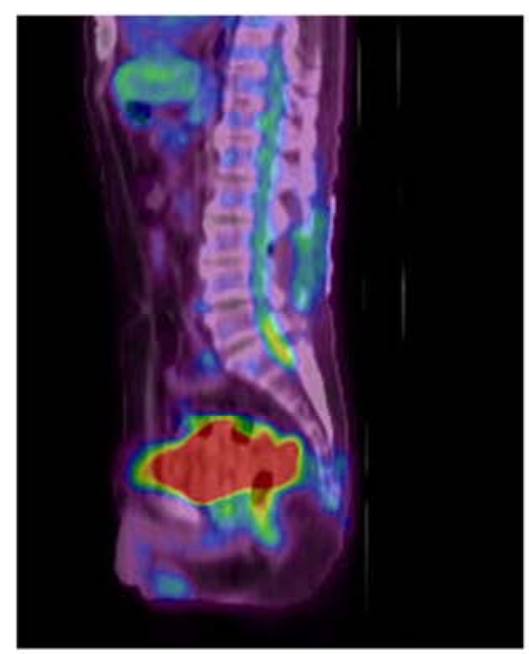

A

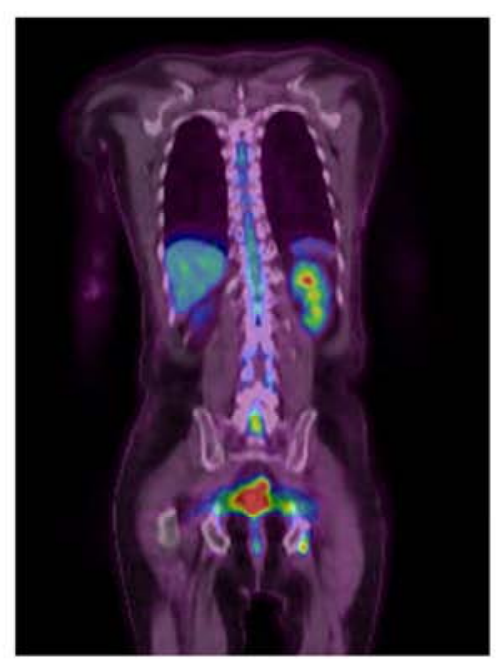

B

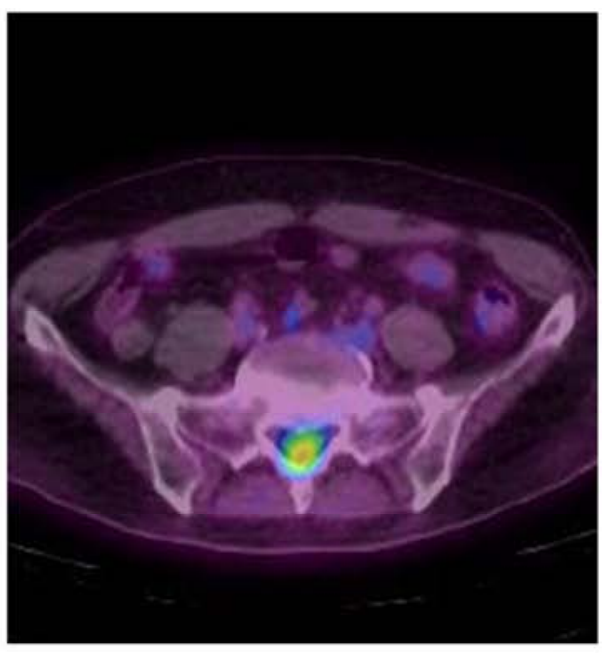

$\mathrm{C}$

Figure 4. Fused PET-CT images showing increased metabolic activity in the spine in sagittal (A), coronal (B) and axial cuts (C, of the lumbar spine).

A whole body PET-CT scan was ordered to rule out any neoplastic activity in other parts of the body. It showed diffuse, subtle leptomeningeal enhancement in the cervical, thoracic and lumbar spinal cord, relatively most intense in the cauda equina region. There were no other areas of abnormally high metabolic activity (Figures 3 and 4).

Because of this finding, laminectomy of L3 and L4 and biopsies of the its arachnoid matter and nerve roots were done. During the procedure, a ventriculo-peritoneal shunt was inserted to address his communicating hydrocephalus and CSF samples were also obtained. CSF glucose was $71 \mathrm{mg} / \mathrm{dl}$ (normal), protein $36.9 \mathrm{mg} / \mathrm{dl}$ (normal), RBC 4 cells per $\mathrm{uL}$, WBC 4 cells per $\mathrm{u} / \mathrm{L}$ which were all lymphocytes. Bacterial culture studies and TB PCR were negative, and histopathology is negative for malignant cells.

However, the arachnoid matter and nerve roots showed atypical mononuclear infiltrates and the leptomeninges had meningothelial cell reaction (Figure 5). The L3 dura is consistent with B-cell non-Hodgkin lymphoma with the following histochemical profile:

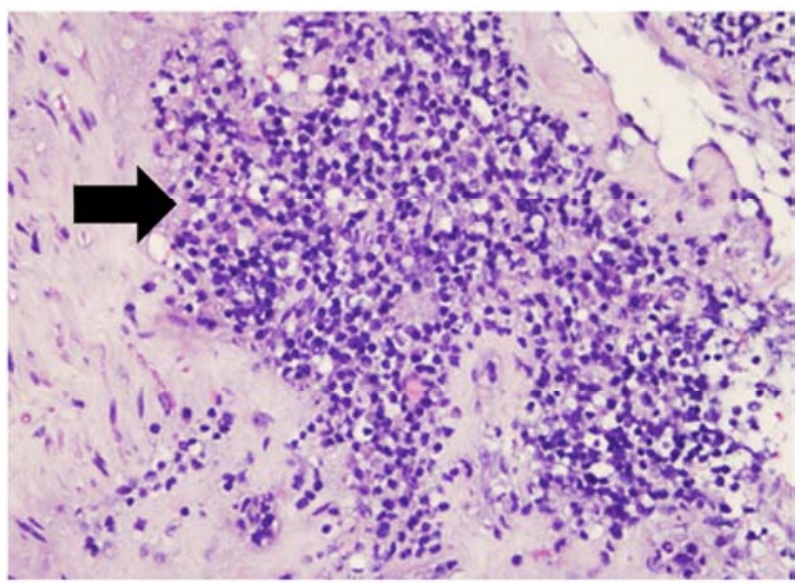

Figure 5. Hematoxylin and eosin stain of patient WDR's lumbar mass showing atypical mononuclear cells (arrow).
CD 20 positive, CD 79a positive, CD3 negative, MUM 1 positive (strong, nuclear), CD 138 negative, CD 68 negative, Kappa positive in polyclonal plasma cells, Lambda positive in polyclonal plasma cells, CD10 negative, BCL 2 positive, Ki67 positive (variable, 5-10\%) (Figures 6A-6E, 7A-7C).

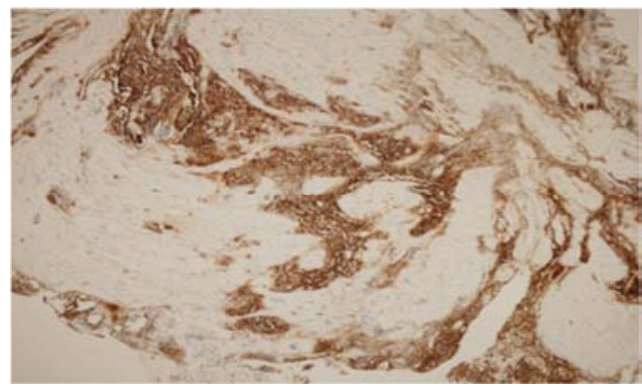

A

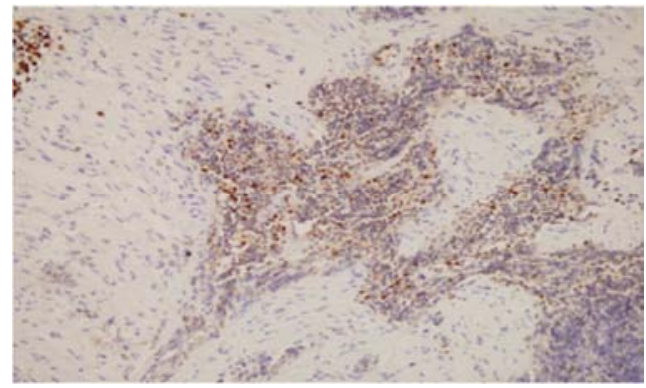

B

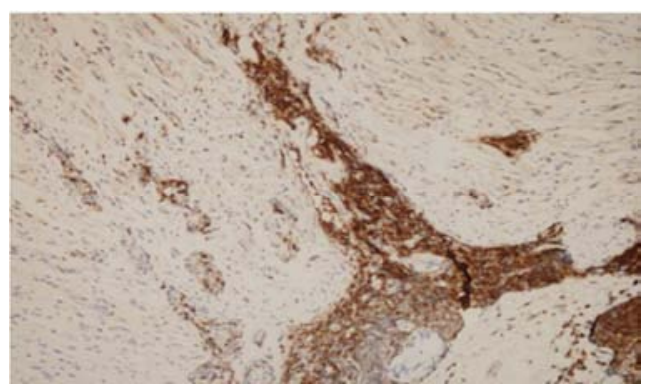

C 


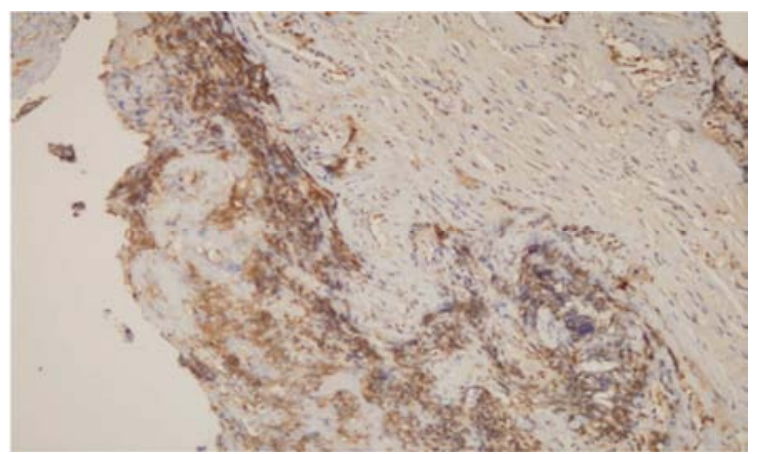

$\mathrm{D}$

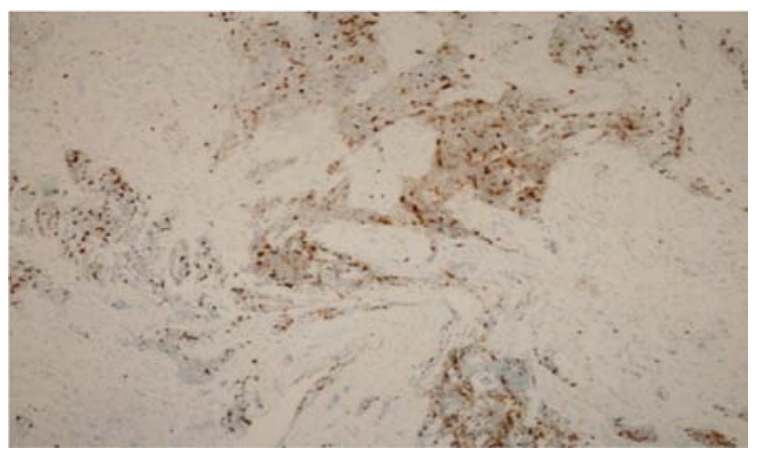

E

Figure 6. Immunohistochemical profile of patient WDR showing positive for CD20, MUM1, Kappa, Lambda and Ki67 (A, B, C, D, E respectively).

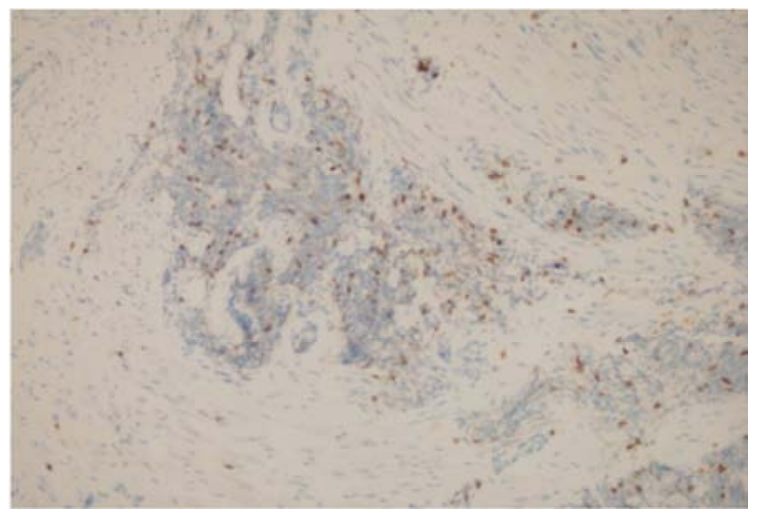

A

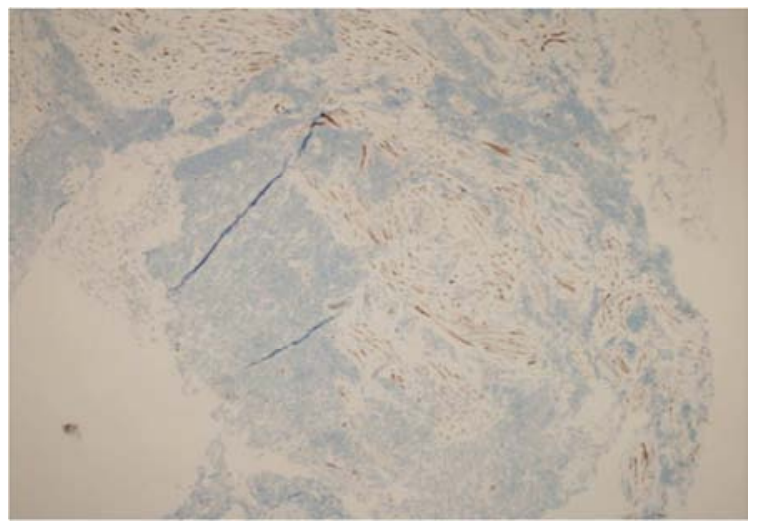

B

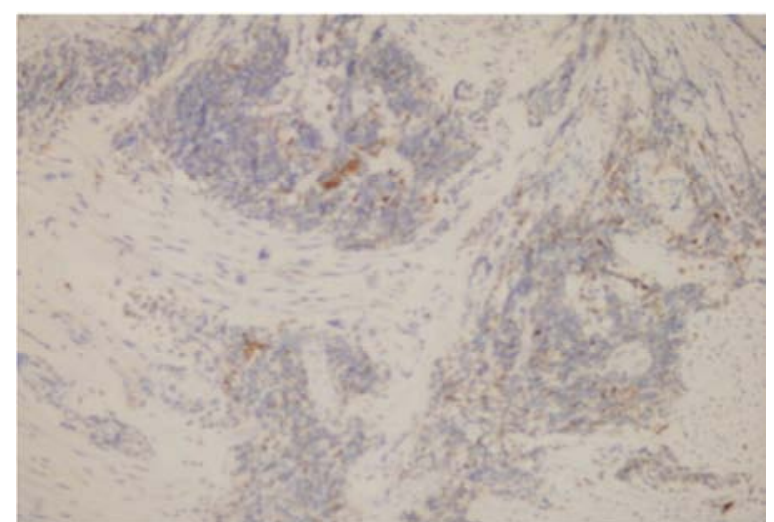

C

Figure 7. Immunohistochemical profile of patient WDR showing negative for $C D 3, C D 10, C D$ 68, (A, $B, C$ respectively).

Post-operatively, his memory and mental agility improved as reported by his relatives. However, he was still moody and irritable. He still preferred to stay in bed or keep seated despite good standing balance and motor strength. Overall, he was still dependent on most activities of daily living.

He was able to complete 6 cycles of high dose Methotrexate and three Rituximab doses. Currently, he still prefers to be wheelchair-bound despite having good motor strength in his extremities. His wife still reports general loss of vigor, occasional loss of recent memory and still had very labile mood. His urinary retention has resolved and is now able to void freely without the use of a catheter. He still has some constipation but now uses laxatives less frequently.

\section{Discussion}

Leptomeningeal carcinomatosis often presents with symptoms of normal pressure hydrocephalus (NPH). It is classically described as the triad of gait disturbances, dementia and urinary incontinence. This explains the behavioral changes, depressive symptoms and gait instability of the patient.

There have been no reports of back pain as its initial presentation. However, two case reports have presented PLL manifesting as multiple radiculopathy in the form of lower extremity weakness $[12,13]$.

The ventriculomegaly persistently seen in his cranial imaging studies prior to VPS insertion is attributed to the impaired CSF absorption due to the inflamed meninges due to the lymphocytic infiltrates. A study done by Omuro et.al observed outcomes of patients with increased intracranial pressure from leptomeningeal carcinomatosis after VPS insertion [14]. Results show improvement of symptoms in $77 \%$ of cases, much like what was observed in this patient. His gait disturbance, as well as urinary retention, may also be explained by the cauda equina syndrome caused by the lymphoma that is prominent on the said area. It is noted that these symptoms resolved after he received chemotherapy. The same mechanism is observed in patients with infectious meningitis. Seizures in patients with leptomeningeal 
pathologies, whether focal or generalized, are due to the meningeal irritation on the cortex. It is possible for patients to have no epileptiform changes on EEG despite the structural cause of seizures.

The hazy appearance of subarachnoid space and the presence of focal seizures swayed his physicians to consider meningitis before CNS malignancies. In the Philippines where tuberculous meningitis is not unusual, this is could be an early sign. Contributory to the suspicion is the xanthochromic appearance of CSF as well as the lymphocytic predominance and very high protein. His CSF AFB showed negative results but this test only shows a specificity of $10-12 \%$ and so a negative result does not rule out its possibility [15]. Most practitioners would just treat for it empirically rather than miss a diagnosis since the medicines are easily available and the side effects are tolerable while the complications of untreated tuberculous meningitis are fatal. This is what actually happened in this case. However, an infectious process in the CNS is unlikely for this case because of the chronicity of symptoms, which is more characteristic of a malignant process. The patient's immune status and age are non-contributory to the suspicion of an infectious or malignant process.

The definite diagnosis of a leptomeningeal lymphoma was made after biopsy of the dura. The histochemical profile of being CD20 and CD79a positive, and CD3 negative supports

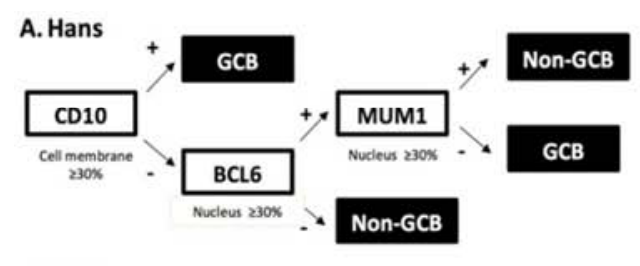

C. Tally
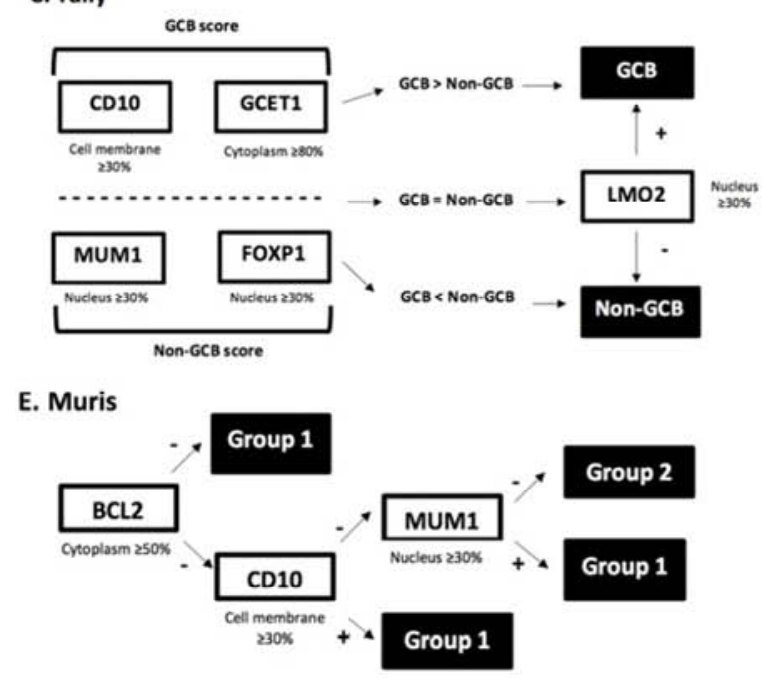

the diagnosis of B-cell clonal proliferation. Positivity to MUM1 and BCL2 points to a probable Diffuse Large B-Cell Lymphoma (DLBCL). This type usually has high Ki-67 $(>50 \%)$ but the low values in the patient's mass may be attributed to steroid use prior to biopsy.

Gene expression profiling (GEP) defines at least three distinct groups of DLBCL that are somewhat predictive of prognosis, namely, germinal center B-cell-like (GCB), activated $\mathrm{B}$-cell-like $(\mathrm{ABC})$ and primary mediastinal large B-cell lymphoma (PMLBCL). The GCB type is believed to have come from germinal centers of $B$ cells while $A B C$ type are from B-cells that have arrested their development into becoming plasma cells. GCB type carries a better prognosis than the ABC type [16].

Not all centers are equipped to do GEP and it is impractical to do so in all specimens for biopsy. In lieu of this, immunohistochemical profile of tumors are obtained and plugged in into algorithms that are used to classify DLBCL into either GCB or ABC. Various algorithms (Figure 8) are of use to clinicians such as those by Hans, by Choi, by Visco-Young, by Tally, by Muris and by Nyman. A study by Hwang et. al compared these algorithms and it showed good concordance with actual survival after the treatment using the R-CHOP protocol (rituximab, cyclophosphamide, adriamycin, vincristine and prednisone) [17].
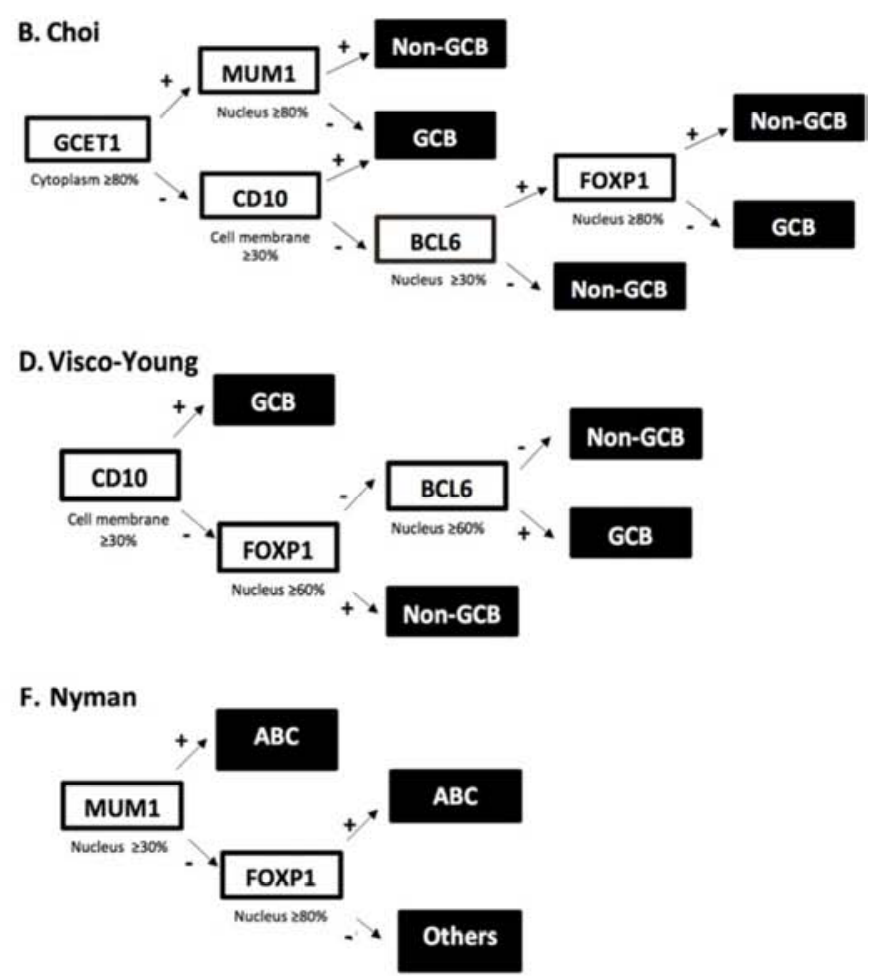

Figure 8. Image lifted from Hwang et.al (High concordance of Gene Expression Profiling-correlated Immunohistochemistry Algorithms in Diffuse Large B-cell Lympoma, not otherwise specified, 2014) showing the different immunohistochemical algorithms used to classify DLBCL into either GCB or ABC: (A) Hans, (B) Choi, (C) Tally, (D) Visco-Young, (E) Muris and (F) Nyman. "Group 1" in (E) Muris algorithm correspond to the GCB type while "Group 2" is to the ABC type.

Given patient WDR's available immunohistochemical profile of being BCL2 positive, CD10 negative and MUM1 positive, he could be classified into DLBCL ABC-type on the Muris or Nyman algorithms. This gives a possibility of a poorer prognosis.

However, for PCNSL or PLL, high dose methotrexate with or without radiotherapy is the mode of treatment [6]. MacNealy et.al described a case where intra-arterial 
methotrexate combined with intravenous etoposide and IV cyclophosphamide was used after reversible blood brain barrier disruption. The patient survived and remained disease-free for 34 months before succumbing to end-stage lymphoma in the adrenal gland and thigh [18]. Therefore, the utility of using the immunohistochemical algorithms mentioned above may not be applicable in classifying and prognosticating DLBCL in the leptomeninges.

Healthcare costs in the Philippines is still mostly out of pocket [19] and in a developing country like the Philippines, it is fortunate that he was able to avail of healthcare and a complete array of diagnostic tests when needed. This poses a challenge to practitioners who would encounter similar cases to choose and prioritize diagnostic examinations needed to arrive at a rare diagnosis such as PLL.

This report is limited by the chronicity of the symptoms and some recall bias of the informant. A detailed neurologic examination at each point in the course is not available to the authors.

\section{Conclusion}

Primary Leptomeningeal Lymphoma is a rare diagnosis presenting with non-specific symptoms, such as dementia and low back pain in this case. A patient's immune status is non-contributory but elderly patients are more prone to this malignancy. A high index of suspicion must be exercised when the presenting case is atypical for meningitis, dementia or other neurologic or non-neurologic disease entities. PLL may present with symptoms indirectly associated with the disease such as gait disturbances, dementia and urinary incontinence (NPH), neck pain, back pain, headache, etc. Imaging studies may mimic that of meningitis as it also presents with dural enhancement and hydrocephalus. These can be used as bases to empirically treat for an infectious etiology in a developing country like the Philippines where diseases such as tuberculous meningitis are not uncommon.

One method of diagnosing the disease is through lumbar puncture and to send for CSF histopathology. However, if CSF studies yield inconclusive results, a dural biopsy may be done to confirm the diagnosis. The immunohistochemical profile of the tumor may help in predicting tumor behavior and prognostication.

Other studies such as CT, MRI or PET CT may be done to look for malignancies in other parts of the body prior to committing to a diagnosis of a primary leptomeningeal lymphoma. Treatment for PLL is still controversial but high dose Methotrexate, radiotherapy or a combination of both is acceptable in current practice.

\section{References}

[1] Ropper A. H., Samuels M. A., Klein J. P. Adams and Victor's Principles of Neurology. $10^{\text {th }}$ ed. New York: Mc-Graw-Hill Educational Medical; 2009.
[2] Fischer, L., Martus, P., Weller, M., Klasen, H.-A., Rohden, B., Röth, A.,... Korfel, A. (2008). Meningeal dissemination in primary CNS lymphoma. Neurology, 71, 1102-1108.

[3] Lachance, D. H., O’Neill, B. P., Macdonald, D. R.,... Posner J. B. (1991) Primary leptomeningeal lymphoma: report of 9 cases, diagnosis with immunocytochemical analysis, and review of the literature. Neurology. Jan; 41 (1): 95-100.

[4] Taylor, J. W., Flanagan E. P., O’Neill B. P., Siegal T...Schiff, D. (2013). Primary leptomeningeal lymphoma. Neurology, Nov 5; 81 (10):1690-1696.

[5] Galarza, M., Gazzeri, R., Elfeky, H. a, \& Johnson, R. R. (2006). Primary diffuse large B-cell lymphoma of the dura mater and cranial vault. Case report and literature review. Neurosurgical Focus, 21 (5), E10. Retrieved from http://www.ncbi.nlm.nih.gov/pubmed/17134112.

[6] Iwamoto, F., \& Abrey, L. (2006). Primary dural lymphomas: a review. Neurosurg Focus, 21 (5), 1-5.

[7] Freilich R. J., Krol G., DeAngelis L. M. (1995) Neuroimaging and Cerebrospinal Fluid Cytology in the Diagnosis of Leptomeningeal Metastasis. Ann Neurol. 1995; 38:51-57.

[8] Carlson C. L., Hartman R., Ly J. Q., Beall D. P. (2003) Primary leptomeningeal lymphoma of the lumbar spine. Clin Imaging, 27: 389-393.

[9] Gijtenbeek, J. M. M., Rosenblum, M. K., \& DeAngelis, L. M. (2011). Primary Central Nervous System T-cell Lymphoma. Neurology, 57 (4), 716-718.

[10] Shenkier, T. N., Blay, J., Neill, B. P. O., Poortmans, P., Thiel, E., Jahnke, K., ... Connors, J. M. (2005). Primary CNS Lymphoma of T-Cell Origin: A Descriptive Analysis From the International Primary CNS Lymphoma Collaborative Group. Journal of Clinical Oncology, 23 (10), 2233-2239. http://doi.org/10.1200/JCO.2005.07.109.

[11] Chong, A., Song, H.-C., Byun, B.-H., Hong, S.-P., Min, J.-J., Bom, H.-S., ... Lee, J.-K. (2013). Changes in (18) f-fluorodeoxyglucose uptake in the spinal cord in a healthy population on serial positron emission tomography/computed tomography. Chonnam Medical Journal, 49 (1), 38-42. http://doi.org/10.4068/cmj.2013.49.1.38.

[12] Cappellari, M., Benedetti, F., Fiaschi A., Benedetti M. D. (2011). Multiple radiculopathy as the presenting manifestation of primary spinal leptomeningeal B cell lymphoma detected by flow cytometry of cerebrospinal fluid. Neurol Sci, 32:1171-1174.

[13] Beitzke, M., Enzinger, C., Beitze, D....Fazekas, F. (2010) Primary leptomeningeal lymphoma of the cauda equina: a rare cause of radiculopathy. J Neurol, 257:1734-1737.

[14] Omuro, A. M. P., Lallana, E. C., Bilsky, M. H., \& Deangelis, L. M. (2005). Venticuloperitoneal shunt in patients with leptomeningeal metastasis. Neurology, 64, 1625-1628.

[15] Clarridge, J. E. I., Shawar, R. M., Shinnick, T. M., \& Plikaytis, B. B. (1993). Large-Scale Use of Polymerase Chain Reaction for Detection of Mycobacterium tuberculosis in a Routine Mycobacteriology Laboratory. Journal of Clinical Microbiology, 31 (8), 2049-2056.

[16] Perry, A. M., Mitrovic, Z., \& Chan, W. C. (2012). Biological prognostic markers in diffuse large B-cell lymphoma. Cancer Control, 19 (3), 214-226. 
[17] Hwang, H. S., Park, C., Yoon, D. H., Suh, C., \& Huh, J. (2014). High Concordance of Gene Expression Profiling - correlated Immunohistochemistry Algorithms in Diffuse Large B-cell Lymphoma,. American Journal of Surgical Pathology, 38 (8), $1046-1057$.

[18] MacNealy, M. W. C., Newton, H. B., McGregor, J. M., Bell, S. D., Ray Chaudhury, A., Slone, H. W., \& Bourekas, E. C. (2008). Primary meningeal CNS lymphoma treated with intra-arterial chemotherapy and blood-brain barrier disruption. Journal of Neuro-Oncology, 90 (3), 329-333. http://doi.org/10.1007/s11060-008-9667-5.

[19] Ulep, V., \& dela Cruz, N. A. (2014). Analysis of Out-of-Pocket Expenditures in the Philippines. 